\title{
Collective scattering of massive stars in higher order gravity
}

\author{
D. Hadjimichef \\ Instituto de Física Teórica-UNESP, Rua Pamplona 145, CEP 01405-900, São Paulo, SP, Brazil \\ and Instituto de Física-Universidade de São Paulo, Caixa Postal 20516, 01498-900 São Paulo, SP, Brazil \\ F. Kokubun \\ Instituto de Física Teórica-UNESP, Rua Pamplona 145, CEP 01405-900, São Paulo, SP, Brazil \\ and Departamento de Astronomia, IAG-USP, Caixa Postal 9638, CEP 01065-970 São Paulo, SP, Brazil
}

(Received 19 March 1996)

In the weak field approximation of higher order gravity theory a gravitational potential is described by a Newtonian plus a Yukawa-like term. This new term is used to explain some aspects of galactic dynamics, without considering dark matter. Its presence modifies the scattering probability of a massive intruder star and relaxation time of the stellar system. [S0556-2821(97)05802-5]

PACS number(s): 04.50.+h, 95.35.+d

\section{INTRODUCTION}

One of the great puzzles of modern cosmology is the dark matter problem. Its existence is inferred by analyzing the rotational curve of some spiral galaxies and the dynamics of galactic clusters [1]. With the introduction of dark matter, the discrepancies obtained using only luminous matter can be avoided. Nevertheless, the origin of dark matter and also its properties are not well understood. Although feasible, the dark matter hypothesis demands the existence of yet nondetected particles. Moreover, its nature and properties are a matter of controversy.

Besides dark matter, alternative gravitational theories were proposed by some authors $[2,3]$ in a tentative way to explain these observational discrepancies. The results of these theories applied in astrophysical situations may be used to constrain or even avoid some problems resulting from the use of standard gravitational theory. These modifications range from changing Newton's second law [3] to modifications in general relativity [4-9]. Among several theories proposed as substitutes of general relativity, the very interesting ones are those with non-Newtonian limit in the weak field approximation. In one class of theories a Yukawa-like term is added to standard the Newtonian potential. The introduction of this term in the classical Newtonian gravitational potential has several origins: scale invariance [4], Brans-Dicke theory with finite mass scalar field [5], and generalized Einstein action with quadratic terms $[6,8,9]$. The main reason for developing these theories was the search for possible candidates to a viable quantum gravity. Nevertheless, with the detection of some disturbances in the galactic dynamics, some authors have tried to explain this with nonstandard gravitational theories. In particular, Sanders [2] used a gravitational potential with a Yukawa term to explain some features of galactic rotational curves. The difficulty with these modified theories is that the analysis of the observational data is inconclusive [1]. So the preferred explanation for galactic dynamics is the presence of dark matter. Therefore it would be important to search other observational situations to test the possible existence of a Yukawa-like term in the gravitational potential. In this work we analyze an idealized homogeneous stellar system which is perturbed due to the passage of a massive intruder star. We calculate the influence of the Yukawa term in the scattering of the massive star and the relaxation time of this system. For this purpose, we use an approach developed by Saslaw [10], in which the scattering is described using a collisionless Boltzmann equation. The advantage of this method is that we can perform a fully analytical approach to the subject, and determine the influence of Yukawa-like term in the final results.

\section{DESCRIPTION OF THE SYSTEM AND THE FORMALISM}

We analyze an idealized homogeneous stellar system, with a distribution of stars all with same mass $m$. A massive intruder star is shot into the system with initial momentum $p_{o}$ and its passage induces a slight inhomogeneity which is responsible for its scattering. This inhomogeneity receives the name of "grexon" which is an acronym for "gravitational excitation" [10]. The grexons reflect a collective response of the system to the passage of the intruder star. The intruder star is massive, in the sense that its mass $M_{o}$ is much greater than $m$. The scattering is calculated using the collisionless Boltzmann equation. The central idea is to deduce the evolution equation of the distribution function $f(\mathbf{p}, \mathbf{x})$, in a form suitable to be handled with standard perturbation theory of the Schrödinger equation. For this purpose, the Hamiltonian of the system is written as $H=K+V_{1}(r)+V_{2}(r)$ with $\left|V_{2}(r)\right| \ll\left|V_{1}(r)\right|$, where $K$ is the kinetic energy, $V_{2}(r)$ is the potential due to massive intruder star, and $V_{1}(r)$ the potential due to all other stars, except the massive one. In this way we can consider the $V_{2}$ terms as a kind of perturbation to the initially "unperturbed" system. A standard Boltzmann equation is written as

$$
\frac{\partial f}{\partial t}=\{H, f\},
$$

where $H$ is the Hamiltonian of the system and $f$ is the distribution function. Now, writing $B f \equiv i\{H, f\}$ and $f=\psi^{*} \psi$ with $\psi=e^{i \theta} \phi$, we obtain 


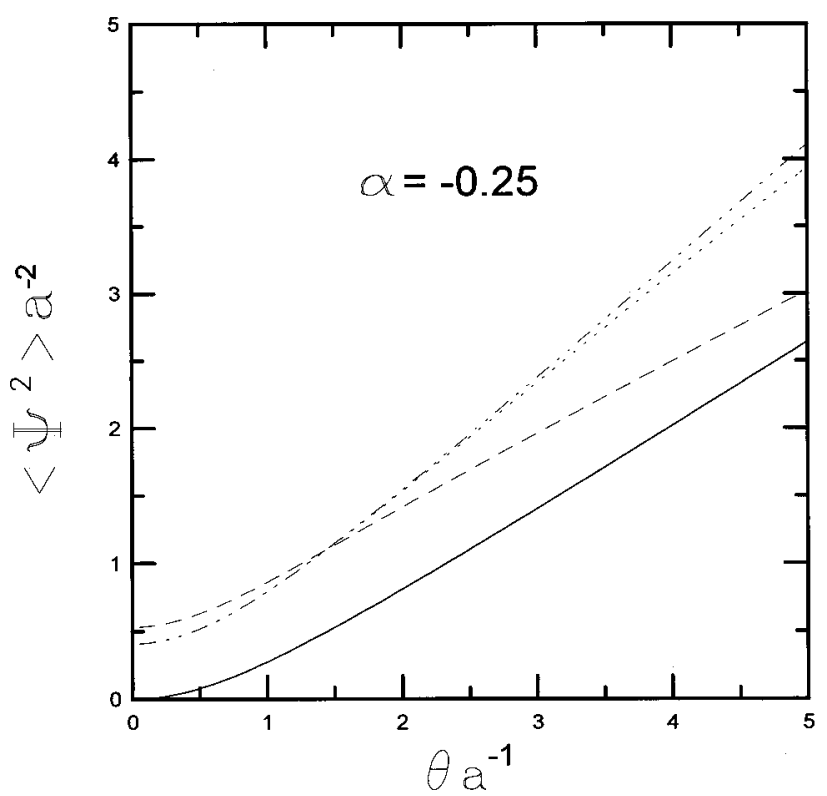

FIG. 1. Mean deflection angle; $\alpha=-0.9, a=0.01$; Newtonian potential (solid line); potential with a Yukawa term: $\sigma=0.5$ (dashdouble-dot), 0.1 (dot), and 0.01 (dash).

$$
i \frac{\partial \phi}{\partial t}=B \phi
$$

which is similar to the Schrödinger equation. Note that from its definition the Poisson bracket operator $B$ is linear and Hermitian.

If one assumes that the initial distribution of stars is homogeneous and time independent, the solution of the above equation can be represented as a plane wave solution $\phi_{o}$ $\propto e^{i \mathbf{k} \cdot \mathbf{r}}$. In this case, the operator $B_{o}$ is given only by kinetic term (the potential is assumed constant) and

$$
B_{o} \phi_{o}=-i \frac{\mathbf{p}}{m} \cdot \frac{\partial \phi_{o}}{\partial \mathbf{r}}=\omega_{k} \phi_{o},
$$

where $\omega_{k}$ is the eigenvalue and $B_{o}$ is an operator which describes the unperturbed system. The introduction of the massive star perturbs the system, so Eq. (2) is equivalent to a Schrödinger equation with a small perturbation:

$$
i \frac{\partial \phi}{\partial t}=\left(B_{o}+\delta B\right) \phi
$$

where

$$
\delta B=i \frac{\partial V_{2}}{\partial \mathbf{r}} \cdot \frac{\partial}{\partial \mathbf{p}}
$$

Therefore we can use the standard Born expansion commonly used in quantum mechanics to solve it. The general solution of the unperturbed system is obtained by using a standard Fourier analysis, considering the plane wave solution

$$
\phi(\mathbf{p}, t)=R^{-3 / 2} \sum_{\mathbf{k}} a_{\mathbf{k}}(\mathbf{p}, t) e^{i\left(\mathbf{k} \cdot \mathbf{r}-\omega_{\mathbf{k}} t\right)},
$$

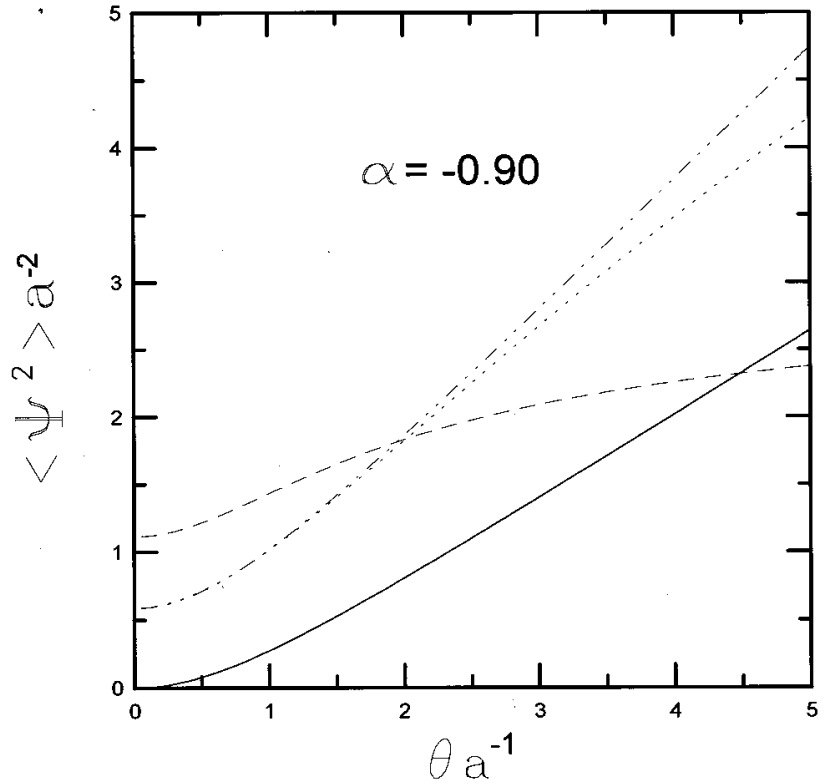

FIG. 2. Mean deflection angle; $\alpha=-0.25, a=0.01$; Newtonian potential (solid line); potential with a Yukawa term: $\sigma=0.5$ (dashdouble-dot), 0.1 (dot), and 0.01 (dash).

where $R$ is the characteristic size of the system, i.e, we are considering the system enclosed in a cubic box of length $R$. The final result will be independent of the size $R$. The only constraint to the $R$ value is that it will be sufficiently large to avoid the influence of surface terms on the final results. In this approach a star is described by the superposition of plane waves with different phases. We need to evaluate the transition matrix

$$
\left\langle\mathbf{k}|\delta B| \mathbf{k}^{\prime}\right\rangle=\int d^{3} r e^{-i \mathbf{k} \cdot \mathbf{r}} \delta B e^{i \mathbf{k}^{\prime} \mathbf{r}}
$$

With this superposition, a given star is described by the group velocity of the resulting wave packet. Working in the limit of small angle scattering, a relation between momentum and wave number of an arbitrary star is [10]

$$
\mathbf{p}=\frac{\left\langle p^{2}\right\rangle}{m \omega_{g}} \mathbf{k}
$$

where $\omega_{g}=\left(4 \pi G \rho_{o}\right)^{1 / 2}$ is the characteristic gravitational response frequency of the system and $\left\langle p^{2}\right\rangle$ is the momentum dispersion.

\section{SCATTERING PROBABILITY AND RELAXATION TIME}

In the framework of a higher order gravity theory, a Yukawa-like term is included in the usual Newtonian potential giving rise to an effective gravitational potential

$$
V=-\frac{G_{\infty} M m}{r}\left(1+\alpha e^{-\mu r}\right) .
$$

The subscript $\infty$ attached to the gravitational constant means the value measured at infinity, i.e., when $\mu r \gg 1$. This fact corresponds to a distance beyond the range of Yukawa term. 
Now, if $\mu^{-1}$ is of the order of galactic sizes, then we measure $G_{\infty}(1+\alpha)$ in laboratory experiments, instead of the usual Newtonian constant $G$. On the other hand, if $\mu^{-1}$ corresponds to distances smaller than the laboratory apparatus, then for any practical purpose the gravitational field is Newtonian and $G \simeq G_{\infty}$. The only appreciable changes would occur at very small scales. Equation (9) results from the weak field approximation of a higher order gravity theory. The introduction of $R^{2}$ and $R_{\mu \nu} R^{\mu \nu}$ in the standard EinsteinHilbert action implies that $\alpha$ will always be positive $[6,8,9]$. Nevertheless, when Eq. (9) is applied to explain galactic rotational curves, it is important to use a negative $\alpha$ value [2], and this can be reached by the introduction of scalar and vector fields $[11,12]$.

To calculate the scattering probability, one needs a Fourier transform of the potential (9) which is obtained by a straightforward procedure. So the probability $\Delta W$ for the energy and momentum transfer per unit time and solid angle $d \Omega[10]$ is

$$
\frac{\Delta W}{t d \Omega}=\frac{(3 \pi)^{2} M_{o}^{2} m G_{\infty} \omega_{g}^{2} \delta\left(\omega-\omega_{g}\right)}{R^{3}\left\langle p^{2}\right\rangle k^{2}}\left[1+\alpha \frac{k^{2}}{k^{2}+\mu^{2}}\right]^{2} .
$$

The scattering probability rate per unit energy $T(\theta, \omega)$ is given by the momentum integral of Eq. (10):

$$
T(\theta, \omega)=\frac{9 M_{o}^{2} G_{\infty} m^{2} \omega_{g}^{3} \delta\left(\omega-\omega_{g}\right)}{8 \pi\left\langle p^{2}\right\rangle^{2} V_{o}\left[\theta^{2}+\xi^{2}\right]}\left[1+\alpha \frac{\theta^{2}+\xi^{2}}{\theta^{2}+\xi^{2}+\sigma^{2}}\right]^{2},
$$

where $\xi=\Delta E /(2 E), \sigma \equiv \Delta E_{g} \mu /\left(\omega_{g} p_{o}\right), V_{o}$ the initial velocity of the intruder star, and $\Delta E_{g} \sim\left\langle p^{2}\right\rangle / m$ [10], with $T(\theta, \omega)$ normalized to one [10]. The mean square deflection angle is calculated by

$$
\begin{aligned}
\left\langle\Psi^{2}\right\rangle & =\int_{0}^{\theta_{1}} d \theta \theta^{2}\left\{\int_{0}^{\infty} d \omega T(\theta, \omega)\right\} \\
& =N\left(\theta_{1}\right)\left[(1+\alpha)^{2} \theta_{1}+\frac{\alpha^{2} \sigma^{2} \theta_{1}}{2\left(a^{2}+\sigma^{2}+\theta_{1}^{2}\right)}-a \arctan \left(\frac{\theta_{1}}{a}\right)-\frac{\alpha}{2 \sqrt{a^{2}+\sigma^{2}}}\left(4 a^{2}+2 a^{2} \alpha+4 \sigma^{2}+3 \alpha \sigma^{2}\right) \arctan \left(\frac{\theta_{1}}{\sqrt{a^{2}+\sigma^{2}}}\right)\right],
\end{aligned}
$$

where $a \equiv \Delta E_{g} /(2 E), \theta_{1}$ is the largest scattering angle associated with this process, and $N\left(\theta_{1}\right)$ is the normalization factor

$$
\frac{1}{N\left(\theta_{1}\right)}=-\frac{\alpha^{2} \sigma^{2} \theta_{1}}{2\left(a^{2}+\sigma^{2}\right)\left(a^{2}+\sigma^{2}+\theta_{1}^{2}\right)}+\frac{1}{a} \arctan \left(\frac{\theta_{1}}{a}\right)+\frac{\alpha}{2\left(a^{2}+\sigma^{2}\right)^{3 / 2}}\left(4 a^{2}+2 a^{2} \alpha+4 \sigma^{2}+3 \alpha \sigma^{2}\right) \arctan \left(\frac{\theta_{1}}{\sqrt{a^{2}+\sigma^{2}}}\right) .
$$

In order to obtain a rough estimate of $\theta_{1}$, it is important to note that the scattering is essentially a binary process, thus in each collision a star is deflected through an angle which is given by $\theta \simeq 2 G M_{o} m /\left(R V_{o}^{2}\right.$ ) (in binary collisions $\mu R \ll 1$, then a Newtonian potential is a good approximation). In the uncorrelated case the massive star scatters $\sqrt{N}$ stars, such that the resulting deflection angle is

$$
\theta_{1} \simeq N^{-1 / 2}\left(\frac{v_{*}}{V_{o}}\right)^{2}
$$

where $v_{*}$ is the dispersion velocity of the system in the Newtonian approximation [in Eq. (A2) we show an exact $v_{*}$ obtained in the weak field limit of higher order gravity]. When scattering is correlated, its is important to consider the number of grexons and their distribution. An estimate of $\theta_{1}$ can be obtained by simply replacing $N$ with number of grexons $N_{g}$. In any of the cases, $\theta_{1}$ is a small angle, which is in agreement with our approach.

In Fig. 1 we plot $\left\langle\Psi^{2}\right\rangle a^{-2} \times \theta_{1} a^{-1}$ and use $a=0.01$, which corresponds to a transfer of $0.5 \%$ of energy between the intruder star and the system for $\alpha=-0.90$ [2] and values $\sigma=0.5$ (dash-double-dot), 0.1 (dot), and 0.01 (dash) representing three distinct situations. The Appendix shows some details of how the parameters $a$ and $\sigma$ are fixed. In the graphs, the solid line shows the result of a pure Newtonian potential [10]. Note that in this case, we are not assuming any particular value for $\mu$ and $R$ (however, note that $\mu$ and $R$ are embodied in the definition of $\sigma$ ). To analyze the effect of an $\alpha$ variation, in Fig. 2 we plot the same set of $\sigma$ as used before with $\alpha=-0.25$ [11]. All curves increase smoothly, but with values of the scattering angle which are different from the Newtonian case. When $\sigma=0.01$ and $\alpha=-0.90$, the curve flattens at the end of $\theta a^{-1}$ axis exhibiting a kind of saturation. For cases $\sigma=0.1$ and 0.01 , see the Appendix. It is important to note that when $\theta_{1} \rightarrow 0$, we obtain the right behavior for $\left\langle\Psi^{2}\right\rangle$, i.e., it goes to zero in this limit. In the figures, this behavior is not clear because the scattering angle rises suddenly near $\theta_{1} \simeq 0$ in the presence of a Yukawa term. Although interesting, the previous results are difficult to be compared with observational data, basically because we do not know how to measure the scattering angle in a stellar system. Therefore it would be important to search for another measurable characteristic. 
The presence of any modifications in the Newtonian potential changes the dynamics of the system, in particular its stability. A parameter related to the stability and more suitable to be compared with observational data is the relaxation time $\tau$ defined as $\tau \equiv E / \dot{E}$, where $E$ is the energy of the intruder star. If $\Delta E$ is the energy transferred in each collision, the total energy transfer is given by

$$
\dot{E}=\int d \Omega \int_{0}^{\infty} d(\Delta E) \Delta E T(\theta, \omega) .
$$

If one assumes that the scattering angle is small, $2 \pi \sin \theta d \theta \simeq 2 \pi \theta d \theta$ and performing the previous integration from $\theta=0$ to $\theta=\theta_{\max }$ - where $\theta_{\max }$ is the angle in which occurs a maximum energy transfer, i.e., the contributions of the collisions with $\theta>\theta_{\max }$ are very small [see Eq. (A5) in the Appendix] — we obtain

$$
\dot{E}=\frac{9 M_{o}^{2} G_{\infty} \omega_{g}^{2}}{8 V_{o}} \lambda,
$$

where

$$
\begin{aligned}
\lambda= & \ln \left(1+\frac{\theta_{\max }^{2}}{a^{2}}\right)+2 \alpha \ln \left(1+\frac{\theta_{\max }^{2}}{a^{2}+\sigma^{2}}\right) \\
& +\alpha^{2}\left[\ln \left(1+\frac{\theta_{\max }^{2}}{a^{2}+\sigma^{2}}\right)-\frac{\theta_{\max }^{2} \sigma^{2}}{\left(\sigma^{2}+\theta_{\max }^{2}+a^{2}\right)\left(a^{2}+\sigma^{2}\right)}\right] .
\end{aligned}
$$

Then one finds that

$$
\tau=\frac{V_{o}^{3} \lambda^{-1}}{9 \pi G_{\infty}^{2} M_{o} m n},
$$

with $n$ the stellar number density. Now we can compare the relaxation time $\tau$ with the Newtonian result obtained by Saslaw [10], which we denote $\tau^{N}$. We obtain after a straightforward calculation that (where we tacitly assumed that $\theta_{\max }$ is the same in both cases)

$$
\begin{aligned}
\frac{\tau^{N}}{\tau}= & \frac{G_{\infty}^{2}}{G^{2}}\left\{1+\frac{2 \alpha}{\ln \left(1+\theta_{\max }^{2} / a^{2}\right)} \ln \left(1+\frac{\theta_{\max }^{2}}{a^{2}+\sigma^{2}}\right)\right. \\
& +\frac{\alpha^{2}}{\ln \left(1+\theta_{\max }^{2} / a^{2}\right)}\left[\ln \left(1+\frac{\theta_{\max }^{2}}{a^{2}+\sigma^{2}}\right)\right. \\
& \left.\left.-\frac{\theta_{\max }^{2} \sigma^{2}}{\left(\sigma^{2}+\theta_{\max }^{2}+a^{2}\right)\left(a^{2}+\sigma^{2}\right)}\right]\right\} .
\end{aligned}
$$

We perform an analysis in three limiting ranges. Let $R_{\text {lab }}$ be a characteristic size of a laboratory (here laboratory ranges from usual terrestrial laboratory to solar system scale). In the first case, $R_{\mathrm{lab}} \ll R \ll \mu^{-1}$, then $G=G_{\infty}$ in all ranges of interest and one finds that

$$
\tau^{N} \simeq \tau .
$$

In the second case, $R_{\text {lab }} \ll \mu^{-1}<R$, and one obtains an interesting result

$$
\tau^{N} \simeq(1+\alpha)^{-2} \tau .
$$

With $-1<\alpha<0$, this corresponds to $\tau^{N}>\tau$, which means that a pure Newtonian system is more stable than a system with a Yukawa term (we are assuming that this relaxation time is dominant in respect to other characteristic stabilization times). Therefore the age of a stellar system in the presence of Yukawa term with range larger than its characteristic size, can be reduced drastically. Note that if $\alpha=-0.9$ this corresponds to $\tau^{N} \simeq 10^{2} \tau$, while if $\alpha=-0.25$ we find $\tau^{N} \simeq 1.78 \tau$. The last case $\mu^{-1} \ll R_{\text {lab }} \ll R$ corresponds to a very small range where one suspects that higher order gravity effects should not be important in astrophysical contexts and measures will not differ from Newtonian results.

\section{CONCLUSION}

In the framework of a higher order gravity theory, which means the introduction of a Yukawa-like term in the gravitational potential, the scattering probability and the relaxation time of the system are modified. A typical size of a globular cluster is about $10 \mathrm{pc}$; therefore we need to have $\mu^{-1}<10$ pc. If Sanders' results are right, globular cluster dynamics would be insensitive to the presence of Yukawalike term. However at galactic clusters scale, its role may be appreciable so the results obtained in this paper may be applied to these systems instead of globular clusters.

Under suitable conditions a system possessing a gravitational potential with higher order terms is more stable than others which do not possess them. If we infer the age of a given system using its dynamical evolution with a Yukawa term its age would be less than in a pure Newtonian system. This fact can be used to constrain the $\alpha$ value using an approach other than measuring rotational curves of galaxies. An important question to be analyzed is the $\alpha$ value. In a higher order gravity coupled to scalar and vector fields, the $\alpha$ value is negative, but its absolute value is less than the expected one. Sanders obtained $\alpha$ in the range $-0.90>\alpha>-0.95$, whereas $\alpha \simeq-0.25$ in the presence of scalar and vector fields [11].

\section{ACKNOWLEDGMENTS}

F.K. thanks the participants of the XXI Annual Meeting of the Brazilian Astronomical Society, with special thanks to J. Horvath, E.M. de Gouveia Dal Pino, G. Medina-Tanco, and R. Opher for comments and suggestions. This work was supported by CAPES, CNPq (D.H.), and FAPESP (F.K.), Brazilian Financial agencies.

\section{APPENDIX}

\section{Estimation of the energy transfer parameter $a$}

In order to evaluate $a=\Delta E_{g} / 2 E$ we use the approximation $\Delta E_{g} \sim \dot{E} R / V_{o}$. So one finds that $a \simeq(\dot{E} R) /\left(2 E V_{o}\right)$. By means of the definition of the relaxation time $\tau=E / \dot{E}$, Eqs. (16) and (17) and the relation $n=3 N /\left(4 \pi R^{3}\right)$, we obtain

$$
a \simeq \frac{27}{8} \frac{G_{\infty}^{2} M_{o} N m}{R^{2} V_{o}^{4}} \lambda .
$$


TABLE I. Some values of $\beta$ and $\kappa$ with $\mu R=1.50$ and $a=0.01$

\begin{tabular}{lccc}
\hline \hline$\alpha$ & $\sigma$ & $\kappa \geqslant$ & $\beta \geqslant$ \\
\hline \multirow{4}{*}{0.90} & 0.5 & 1.4 & 1.2 \\
& 0.5 & 5.0 & 0.3 \\
& 0.1 & 1.4 & 5.7 \\
& 0.1 & 5.0 & 1.6 \\
& 0.01 & 1.6 & 50 \\
& 0.01 & 5.0 & 16 \\
\hline 0.25 & 0.5 & 1.4 & 1.2 \\
& 0.5 & 5.0 & 0.3 \\
& 0.1 & 1.4 & 5.8 \\
& 0.1 & 5.0 & 1.6 \\
& 0.01 & 1.6 & 49 \\
\hline \hline
\end{tabular}

From virial theorem one has that the kinetic energy $K$ can be expressed as $K=(1 / 2) \vec{r} \cdot \vec{F}$, where $F=-\vec{\nabla} V$ and $V$ is the potential defined by Eq. (9), so one can obtain the dispersion velocity

$$
v_{*}^{2}=\frac{G_{\infty} N m}{R}\left[1+\alpha(1+\mu R) e^{-\mu R}\right] .
$$

The parameter $a$ can now be given by

$$
a \simeq \frac{27}{8} \frac{\lambda}{N} \frac{\beta}{\kappa^{4}} \frac{1}{\left[1+\alpha(1+\mu R) e^{-\mu R}\right]^{2}},
$$

where $\beta$ and $\kappa$ are the mass and velocity ratios

$$
\beta=\frac{M_{o}}{m}, \quad \kappa=\frac{V_{o}}{v_{*}} .
$$

We can now draw some conclusions about $a$, which basically describes the influence of intruder star on the scattering system. If $a$ is large, essentially $\beta / \kappa^{4}$ is large too. In this case, we would have a strong perturbation of the intruder on the system, in such a way that our approach breaks down. On the other hand, for a small $a$ the resulting interaction of the intruder on the system is small. These considerations are important for fixing the $a$ value and for this purpose $\lambda$ should be estimated. We use the relation

$$
\frac{\theta_{\max }}{a}=\frac{k_{\max } V_{o}}{\omega_{g}}
$$

for evaluating $k_{\max } V_{o} / \omega_{g}$, which is considered in the uncorrelated and correlated scattering situations (a more detailed exposition can be found in [10]). In the uncorrelated case, $k_{\max }$ is of the same order as the inverse of the distance between the stars, i.e., $k_{\max } \simeq N^{1 / 3} R^{-1}$, then

$$
\frac{k_{\max }^{2} V_{o}^{2}}{\omega_{g}^{2}} \simeq N^{2 / 3} \kappa^{2} \gg 1,
$$

where we used the relation $\omega_{g}=\sqrt{4 \pi G \rho_{o}}$, with $\rho_{o}=N m /\left(4 \pi R^{3} / 3\right)$. In the case of correlated scattering, we
TABLE II. Some values of $\beta$ and $\kappa$ with $\mu R=0.5$ and $a=0.01$. (*) The cases with $\kappa<1$ are not desirable, because the chances of the intruder star being captured is high.

\begin{tabular}{lccc}
\hline \hline$\alpha$ & $\sigma$ & $\kappa \leqslant$ & $\beta \leqslant$ \\
\hline \multirow{3}{*}{-0.90} & 0.5 & 1.2 & 1.3 \\
& 0.1 & 2.0 & 4.1 \\
& 0.01 & 2.5 & 32 \\
\hline \multirow{3}{*}{0.25} & 0.5 & $0.62^{*}$ & 2.7 \\
& 0.1 & $0.86^{*}$ & 9.5 \\
& 0.01 & 1.35 & 60 \\
& 0.01 & 1.72 & 48 \\
\hline \hline
\end{tabular}

need to consider the number of "grexons" $N_{g}$ inside the cluster. For example, if its distribution is spherical, we have $k_{\max } \simeq N_{g}^{1 / 3} / R$, such that

$$
\left(\frac{k_{\max } V_{o}}{\omega_{g}}\right)^{2} \simeq N_{g}^{2 / 3} \kappa^{2}>1
$$

In all cases we have $k_{\max }^{2} V_{o}^{2} / \omega_{g}^{2}$ as a dominant term in the logarithm. In the definition of $\lambda$ [Eq. (17)] the first term is dominant, while the others render small corrections. So, we obtain that $\lambda \simeq 1 \sim 10$ with $N \sim 10^{5}$ (a typical number of stars in a globular cluster). Because of the logarithmic dependence, our results do not depend strongly on the exact value of $k_{\max }^{2} V_{o}^{2} / \omega_{g}^{2}$.

\section{Estimation of parameter $\sigma$}

Starting from the definition of $\sigma$,

$$
\sigma \equiv \frac{\Delta E_{g} \mu}{\left(\omega_{g} p_{0}\right)},
$$

and using the relation $\Delta E=\left\langle p^{2}\right\rangle / m$ [10] we obtain that

$$
\sigma^{2}=\frac{\left\langle p^{2}\right\rangle^{2} \mu}{\left(m^{2} \omega_{g}^{2} p_{o}^{2}\right)},
$$

where $\omega_{g}^{2}=4 \pi \widetilde{G} \rho=3 \widetilde{G} N m / R^{3}$, with $N$ the number of stars and $\widetilde{G}$ is the effective gravitational constant, which in the presence of a Yukawa potential can be calculated by perturbation theory, but for our following purposes the results will independent of $\widetilde{G}$. If one now assumes that $p_{o}^{2}=c_{1}^{2}\left\langle p^{2}\right\rangle$ and $\left\langle p^{2}\right\rangle \simeq m^{2} v_{*}^{2}$ where $c_{1} \simeq \kappa \beta$, the following relation is obtained:

$$
\sigma \simeq \sqrt{2 / 3}\left(\frac{R \mu}{c_{1}}\right)
$$

If $R_{\mathrm{lab}} \simeq \mu^{-1} \leqslant R$ one finds that

$$
c_{1} \simeq \kappa \beta \geqslant \frac{1}{\sigma} \sqrt{2 / 3}
$$

such that 


$$
a \geqslant \frac{27}{8} \frac{\lambda}{N \sigma \kappa^{5}} \frac{1}{\left[1+\alpha(1+\mu R) e^{-\mu R}\right]^{2}} .
$$

Now we can estimate $a$ and $\sigma$ for some particular situations. In our case, we are interested in the scattering of a star from a typical globular cluster, so we must have $N \simeq 10^{5}$. From Eq. (A11) we see that the smaller the $\sigma$, the greater the mass of the intruder star (or its velocity with respect to dispersion velocity of the cluster). Two distinct situations can be considered, one is the case in which the mass of the intruder star is nearly the same as the mass $m$ of a star in the cluster. The other is one in which the intruder star is much more massive than $m$. Once we pick up a value for $\sigma$, we constrain $a$. If we consider a $\sigma$ variation in the range determined by the values $0.5,0.1$, and 0.01 we have the possibility of studying a two order of magnitude mass difference.
In Table I we show several sets of $\beta, \kappa$ with $\lambda=10, a=0.01, N=10^{5}$, and $\sigma=0.5,0.1$, and 0.01 in all cases; we set $\mu R=1.50$. Although we always work with $\mu R \geqslant 1$ due to behavior of relaxation time (21) we include, for completeness, in Table II the results when we assume that $\mu R<1$ (more specifically $\mu R=0.5$ and the same $\lambda, a$, and $N$ as in the previous case). This implies changing $\geqslant$ by $\leqslant$ in Eqs. (A11) and (A12).

An important feature is that with $\alpha=-0.25$, there is no way to satisfy the previous constraints with $\sigma=0.5$ and 0.1 . In both cases, we obtain $\kappa<1$, meaning that the velocity of the intruder star is less than the dispersion velocity of the system. In this case, the chances of the intruder star being captured is very high, which is an undesirable feature.
[1] V. Trimble, Annu. Rev. Astron. Astrophys. 25, 425 (1987).

[2] R.H. Sanders, Astron. Astrophys. 136, L21 (1984).

[3] M. Milgrom, Astrophys. J. 270, 365 (1983).

[4] Y. Fujii, Nature (London) 234, 5 (1971); Y. Fujii, Ann. Phys. (N.Y.) 69, 494 (1972).

[5] R. Acharaya and P.A. Hogan, Lett. Nuovo Cimento 6, 668 (1973)

[6] M. Kenmoku, E. Kitajima,Y. Okamoto, and K. Shigemoto, Int. J. Mod. Phys. D 2, 123 (1993).

[7] T. Goldman, J. Pérez-Mercader, Fred Cooper, and Michael
Martin Nieto, Phys. Lett. B 281, 219 (1992).

[8] K.S. Stelle, Phys. Rev. D 16, 953 (1977); Gen. Relativ. Gravit. 9, 353 (1978).

[9] R. Utiyama, Prog. Theor. Phys. 72, 83 (1984).

[10] W.C. Saslaw, Astrophys. J. 195, 773 (1975); W.C. Saslaw, Gravitational Physics of Stellar and Galactic Systems (Cambridge University Press, Cambridge, England, 1987).

[11] M. Kenmoku, Y. Okamoto, and K. Shigemoto, Phys. Rev. D 48, 578 (1993).

[12] F. Kokubun, Mod. Phys. Lett. A 11, 2409 (1996). 